\section{Tabakentwöhnung in der Schweiz}

\author{
Empfehlungen einer Expertengruppe*
}

J. Cornuz

\section{Zusammenfassung}

Das Bundesamt für Gesundheit (BAG) hat die Unité de prévention de Lausanne (eine Gemeinschaftsinstitution des Institut universitaire de médecine sociale et préventive, der Policlinique Médicale Universitaire und des Centre Hospitalier Universitaire Vaudois von Lausanne) mit der Bildung einer Expertengruppe im Bereich Tabakkonsum in der Schweiz beauftragt. Die wichtigsten Empfehlungen dieser Expertengruppe sind hier kurz zusammengefasst:

1. Die Rauchenden dazu bringen, dass sie in der Entwicklung zu Nichtrauchenden weiterkommen. Es geht vor allem darum, die Rauchenden im Stadium "Unentschiedenheit» in das Stadium "Abwägen" zu bringen und zu verhindern, dass Personen, die das Rauchen gerade erst aufgegeben haben, wieder damit anfangen.

2. Den Wandel der Verhaltensnormen bei der Schweizer Bevölkerung im Umgang mit dem Tabak unterstützen.

3. Die Kostenübernahme durch die Krankenkassen für die Einzelberatung, die pharmakologische Unterstützung (Nikotinsubstituten, Bupropion) und die nicht pharmakologische Unterstützung (Einzel- oder Gruppensitzungen) zur Tabakentwöhnung durchsetzen.

\footnotetext{
* Mitglieder der Expertengruppe

Prof. Th. Abelin, Institut für Sozial- und Präventivmedizin, Universität Bern; CIPRET-Bern (ehemals), Progef, Bern; Prof. F. Gutzwiller, Institut für Sozial- und pour la Prévention du Tabagisme, Union internationale contre le cancer, Hôpital Lungenliga Schweiz, Bern, und Policlinique Médicale Universitaire, Lausanne

Vertreterinnen und Vertreter der Auftraggeber

BAG: Frau B. Caretti, FMH: Dr. med. J.-A. Dubois und Dr. med. U. Steiner

Korrespondenz:

PD Dr. med. Jacques Cornuz, MER, MPH

Centre Hospitalier Universitaire Vaudois

Policlinique médicale universitaire und

Institut universitaire de médecine sociale et préventive

Unité de prévention

Rue du Bugnon 21

CH-1005 Lausanne

E-Mail: jacques.cornuz@chuv.hospvd.ch
} Frau V. El Fehri, Arbeitsgemeinschaft Tabakprävention Schweiz, Bern; Frau C. Gafner, Präventivmedizin, Universität Zürich; Prof. A. Hirsch, Präsident des Réseau Européen Saint Louis, Paris, France; Dr. J.-Ch. Rielle, CIPRET-Genève; Dr. med. J.-P. Zellweger,
4. Entwicklung einer Methode zur langfristigen Erforschung der Prävalenz des Tabakkonsums und der tabakbedingten Probleme.

\section{Einleitung}

Der Tabakkonsum stellt in der Schweiz ein schwerwiegendes Problem der öffentlichen Gesundheit dar. Mit einem Anteil von 30\% Rauchenden in der Bevölkerung, das sind 1,7 Millionen Personen, ist die Prävalenz sehr hoch [1]. Es ist eindeutig erwiesen, dass der Tabakkonsum, bedingt durch tabakverursachte Lungen-, Herzkreislauf- und neoplastische Krankheiten, die Hauptursache für vorzeitige Mortalität und Morbidität ist [2, 3]. Umgekehrt lassen sich die tabakbedingte Morbidität und Mortalität sowie auch die allgemeine Mortalität mit dem Rauchstopp reduzieren [4, 5]. Ungeachtet des Gesundheitszustands des Rauchenden (an tabakbedingtem Leiden erkrankt bzw. nicht erkrankt) müssen im Rahmen einer Strategie zur Tabakentwöhnung Präventivmassnahmen getroffen werden [6].

Das Bundesamt für Gesundheit (BAG) hat vor einiger Zeit die Unité de prévention de Lausanne (eine Gemeinschaftsinstitution des Institut universitaire de médecine sociale et préventive und der Policlinique Médicale Universitaire de Lausanne) mit der Bildung einer Expertengruppe im Bereich Tabakkonsum in der Schweiz beauftragt.

Die Experten und Expertinnen dieses Gremiums wurden so ausgewählt, dass die verschiedenen Aspekte der Förderung des Rauchstopps vertreten sind. Bei der Wahl der Experten war ihr Engagement bei der Förderung der Kontrolle des Tabakkonsums in der Schweiz oder in Europa entscheidend. Ausgewählt wurden die Expertinnen und Experten vom Verfasser dieses Berichts mit dem Einverständnis des BAG. Die Auswahl beruht auf zum Teil subjektiven Kriterien. Dies erklärt, warum andere Expertinnen und Experten in der Schweiz nicht berücksichtigt wurden. Die Expertengruppe hat im ganzen fünfmal getagt. Im Verlaufe des Jahres 1999 wurde das Schlussdokument erstellt, das von allen Mitgliedern der Gruppe, und im Verlaufe des Jahres 2000 auch vom BAG, genehmigt wurde. Die wichtigsten Ergebnisse dieses Schlussdokuments sind hier kurz zusammengefasst:

Die Expertengruppe hatte die Aufgabe, für die Schweizerischen Gesundheitsbehörden im allgemeinen und das BAG im besonderen Richtlinien zur Förderung der Tabakentwöhnung auszuarbeiten. Die Gruppe hatte insbesondere die Resultate von zwei Studien zu berücksichtigen, die kürzlich bei in der Schweiz wohnhaften Rauchenden durchgeführt wurden. Die erwähnten Studien wurden 1997 vom Institut für Sozial- und Präventivmedizin Bern und der entsprechenden Präventionseinheit in Lausanne durchgeführt, die erste unter Personen der Allgemeinbevölkerung [7] und die zweite unter den Patientinnen und Patienten der Policlinique Médicale Universitaire de Lausanne [8]. Hauptziel der Studien 
war es, die Rauchenden aufgrund ihrer Rauchgewohnheiten, ihrem Wunsch, mit dem Rauchen aufzuhören, und des Stadiums der Veränderung, in dem sie sich befinden, in Kategorien einzuteilen. Weiter wollte man in Erfahrung bringen, wie die Rauchenden die gesundheitlichen Folgen des Tabakkonsums einschätzen und was sie von der Tabakentwöhnung und der Betreuung beim Versuch, mit dem Rauchen aufzuhören, erwarten.

In den beiden Studien wurden die Rauchenden gemäss dem Modell "Stadien der Veränderung" ("stages of change») [9] in Kategorien eingeteilt. Ein Rauchstopp bedeutet effektiv eine Veränderung des Verhaltens. Dabei handelt es sich um einen dynamischen, stufenweisen Prozess, wie er im Modell "Stadien der Veränderung" beschrieben ist. Rauchende durchlaufen einen Entwicklungszyklus von fünf Motivationsstadien, um aufzuhören. Die Wahrscheinlichkeit, Ex-Rauchende zu werden, nimmt mit jedem durchlaufenen Stadium zu. Nur ein kleiner Teil der Ausstiegsversuche führt zu einer längeren Abstinenz. Normalerweise kommt es zu einem Rückfall und die Rauchenden fallen in eines der drei vorangehenden Stadien zurück. Rauchende durchlaufen diesen Zyklus mehrmals; im Durchschnitt sind 3-6 Ausstiegsversuche nötig, bevor sie Ex-Rauchende werden. Die Anwendung dieses Modells erlaubt es, die Intervention zu individualisieren. Die Intensität und der Inhalt der Behandlung sind dem Motivationsstadium des Aufhörens anzupassen. Selbst wenn der Ausstieg nicht sofort gelingt, verbessert eine gezielte Beratung die Motivation vieler Rauchender, mit dem Rauchen aufzuhören, und somit ihre Chancen, später beim Aufhören erfolgreich zu sein.

\section{Stadien der Veränderung beim Tabakausstieg}

\section{Unentschiedenheit ("precontemplation")}

- Der/die Rauchende denkt nicht ernsthaft daran, das Rauchen in den nächsten sechs Monaten aufzugeben.

- Die Vorteile der Zigarette sind wichtiger als die Risiken des Rauchens.

- Der/die Rauchende ist nicht empfänglich für die traditionellen Präventionsbotschaften ("Rauchen schadet der Gesundheit»).

Abwägen ("contemplation»)

- Der/die Rauchende denkt ernsthaft daran, das Rauchen zwar nicht sofort, aber in den nächsten sechs Monaten aufzugeben.

- Die Haltung des/der Rauchenden ist noch ambivalent: Der langfristige Gewinn eines Ausstiegs ist gleich wichtig wie die momentanen Vorteile des Rauchens.

Vorbereiten ("preparation»)

- Der/die Rauchende ist fest entschlossen, das Rauchen in den nächsten vier Wochen aufzugeben.

- Der Gewinn eines Ausstiegs ist wichtiger als die momentanen Vorteile des Rauchens.
- Der/die Rauchende sucht Hilfe und/oder Beratung.

\section{Handeln ("action»)}

- Der/die Rauchende hat das Rauchen vor weniger als sechs Monaten aufgegeben.

- Die Gefahr eines Rückfalls ist gross.

- Der/die Rauchende unternimmt aktiv Anstrengungen, um Nichtraucher/in zu bleiben.

\section{Konsolidierung ("maintenance»)}

- Der/die Rauchende hat das Rauchen vor mehr als sechs Monaten aufgegeben.

- Die Gefahr eines Rückfalls nimmt ab.

- Der/die Rauchende unternimmt aktiv Anstrengungen, um Nichtraucher/in zu bleiben.

Die wichtigsten Ergebnisse dieser beiden Studien, die in detaillierten wissenschaftlichen Berichten festgehalten sind (BAG), werden hier kurz zusammengefasst:

- Die Mehrheit der Rauchenden will mit dem Rauchen aufhören.

- Im Gegensatz zu den Ländern, in denen die Kontrolle des Tabakkonsums fortgeschritten ist (in einigen angelsächsischen und skandinavischen Ländern), befindet sich in der Schweiz ein grosser Teil der Rauchenden in der Phase der Präkontemplation, d.h. Rauchende, die in den nächsten sechs Monaten nicht ernsthaft an einen Ausstieg denken (80\% in der Studie 1 und $87 \%$ in der Studie 2).

- In der Allgemeinbevölkerung gibt es einen grossen Anteil von Gelegenheitsrauchenden, d.h. Personen, die weniger als eine Zigarette pro Tag rauchen (11\%, was 28\% aller Rauchenden entspricht).

- Bei den Rauchenden, die mit Rauchen aufgehört haben oder versucht haben aufzuhören, haben die beiden Untersuchungen folgendes ergeben:

- die Mehrheit hat keine strukturierten Methoden angewendet (77\%);

- von den Rauchenden, die mehr als 15 Zigaretten pro Tag rauchen, hat eine beachtliche Minderheit (23\%) Hilfe in Anspruch genommen;

- die Hauptgründe für einen Rauchstopp sind das Auftreten von Krankheiten oder allgemeine Sorgen um die eigene Gesundheit.

Nach Abschluss der Arbeiten der Expertengruppe wurden deren Befunde durch neue Studien gestützt [10]. Zudem haben die Expertinnen und Experten auch Erkenntnissen aus der wissenschaftlichen Literatur Rechnung getragen. Daraus geht hervor, dass das medizinische Personal, aber ganz besonders die Ärzteschaft, unbedingt versuchen sollte, die Rauchenden zu beraten und sie beim Ausstieg zu unterstützen [11-13]. Die Mehrheit der Rauchenden möchte mit Rauchen aufhören und befürwortet eine Betreuung durch das medizinische Fachpersonal. Vertreterinnen und Vertreter der Medizinberufe sind eine bevorzugte Anlaufstelle und tragen häufig zur 
Aufhörentscheidung bei. Zahlreiche Studien und Meta-Analysen haben gezeigt, dass eine medizinische Beratung der Rauchenden wirksam ist [14-17]. Selbst wenn die Betreuung der Medizinalperson nicht sofort zu einem Rauchstopp führt, so hilft sie doch vielen Rauchenden, auf eine weitere Stufe der Veränderung zu kommen. Die Zahl der Ausstiegsversuche wird dadurch erhöht [18]. Zudem liefern Nikotinsubstitute zwischen einem und zwei Dritteln des mit Zigaretten inhalierten Nikotins, vermindern die Entzugserscheinungen und erleichtern so den Tabakausstieg. Zurzeit sind in der Schweiz fünf Formen von Nikotinsubstituten erhältlich: Kaugummi, transdermale Hautpflaster, Nasenspray, Microtabs und Inhalator. Eine kürzlich durchgeführte, umfassende MetaAnalyse hat gezeigt, dass die Abstinenzrate nach 6 bis 12 Monaten durch Nikotinsubstitute um 1,7 multipliziert wird. Diese erreicht im Durchschnitt $17 \%$ für die substituierte Gruppe gegenüber 10\% in der "Placebo"-Gruppe. Es gibt keine signifikanten Unterschiede zwischen den vier verschiedenen Formen von Nikotinsubstitution. Eine weitere Möglichkeit im Bereich der pharmakologischen Unterstützung ist das Antidepressivum Bupropion, das auch nicht depressiven Menschen beim Rauchstopp hilft [19]. Es ist seit kurzem in der Schweiz zugelassen.

Andere Methoden der Tabakentwöhnung sind ebenfalls sehr wirksam [20], wie verhaltenstherapeutische Einzel- und Gruppenprogramme sowie Selbsthilfemethoden zum Rauchstopp mit auf die Bedürfnisse des einzelnen zugeschnittenen Botschaften ("self-help»), so etwa das neue schweizerische Programm "Ziel Nichtrauchen»: Die Teilnehmenden erhalten per Post oder Internet individuelle Ratschläge, die auf den Antworten eines von den Teilnehmenden vorgängig ausgefüllten Fragebogens über das Rauchverhalten basieren [21]. Dagegen sind die Methoden der Akupunktur und der Hypnose, für die es wenig qualitativ hochstehende Studien gibt, unwirksam. Die damit erreichte Ausstiegsrate ist vergleichbar mit denjenigen der Gruppen ohne Intervention und den Placebo-Gruppen (Pseudo-Akupunktur) [20]. Die pharmakologische Unterstützung wurde von der Weltgesundheitsorganisation und ihrer Abteilung "Tobacco Free Initiative» klar als vorrangig eingestuft (http://www.who.int).

Ausgehend von den Erkenntnissen in der wissenschaftlichen Literatur, den Ergebnissen dieser beiden Studien und der Erfahrung der einzelnen Mitglieder hat die Expertengruppe vier Hauptziele festgelegt. Für jedes dieser Ziele wurden zudem die zur Erreichung des Ziels notwendigen allgemeinen und spezifischen Massnahmen erläutert. Die Empfehlungen richten sich nur an die in der Schweiz wohnhafte erwachsene Bevölkerung. Die Expertengruppe ist sich bewusst, dass an Jugendliche gerichtete Massnahmen wichtig sind, doch diese Zielgruppe war nicht Teil ihres Auftrags.

\section{Empfehlungen}

Ziel 1

Die Rauchenden dazu bringen, dass sie in der Entwicklung zu Nichtrauchenden weiterkommen. Es geht vor allem darum, die Rauchenden im Stadium "Unentschiedenheit» in das Stadium "Abwägen" zu bringen und zu verhindern, dass Personen, die das Rauchen gerade erst aufgegeben haben, wieder damit anfangen.

Für die Erreichung dieses Ziels sind spezifische, auf die entsprechende Zielgruppe zugeschnittene Massnahmen notwendig.

\section{Die Rauchenden}

Allgemeine Massnahmen:

1. Die Vorteile des Rauchstopps in Erinnerung rufen.

2. Den Wissensstand im Bereich Tabakentwöhnung verbessern.

Spezifische Massnahmen (Information und Sensibilisierung):

- Durchführung von Plakat- und Inseratenkampagnen in den grossen Zeitungen; dabei ist insbesondere den Empfehlungen für eine effiziente Vermittlung der Präventionsbotschaften Rechnung zu tragen [22].

- Hier einige Beispiele weiterer Handlungsmöglichkeiten:

- die gesundheitsschädigenden Folgen des Tabaks in Erinnerung rufen;

- die Gründe und Vorteile des Rauchstopps in Erinnerung rufen (z.B. Verschwinden des Raucherhustens, deutlich verringertes Risiko, an einem Herz-Kreislauf-Leiden zu erkranken);

- in Erinnerung rufen, dass die Behandlung der Nikotinabhängigkeit bei der Tabakentwöhnung wichtig ist;

- gezielt Personen mit anderen kardiovaskulären (z.B. Bluthochdruck) und pulmonalen Risikofaktoren ansprechen;

- die Rauchenden in den Stadien 1 (Unentschiedenheit) und 2 (Abwägen) besonders ansprechen;

- Differenzieren der Botschaften je nach Zielgruppe (Rauchende am Arbeitsplatz, am Wochenende, mit tiefem Einkommen usw.);

- Bekanntmachen von Gegenargumenten zu den gängigen Argumenten, wie sie von den Rauchenden und/oder der Tabakindustrie zur Ermutigung, weiterzurauchen, verwendet werden (z.B. mit dem Text der Broschüren «Ziel Nichtrauchen");

- Die Beteiligung der Krankenkassen an der Tabakentwöhnung fördern, indem man ihnen vorschlägt, ihren rauchenden Mitgliedern auf Anfrage Broschüren zur Tabakentwöhnung (z.B. die Broschüre "Ziel Nichtrauchen») zu verteilen (mittels Informationsbulletins der Kasse).

Für diese Kampagne sollten bereits existierende Kanäle genutzt werden, z.B. der "Tag des Nichtrauchens». 
Das medizinische Personal

Ärzte, Apotheker, Zahnärzte, Pflegepersonal, Physiotherapeutinnen und -therapeuten.

\section{Allgemeine Massnahmen}

1. Förderung der Unterstützung und des Engagements durch das medizinische Personal, insbesondere durch die "minimale Intervention".

2. Das Tabakfachwissen des medizinischen und nicht medizinischen Personals im Sinne einer Vermittlungsstelle nutzen, um die Rauchenden in der Allgemeinbevölkerung zu erreichen.

3. Förderung der Kostenübernahme von Beratungen und der pharmakologischen Unterstützung (Nikotinsubstitute und Bupropion).

\section{Spezifische Massnahmen}

(Ausbildung und Information)

Ärztinnen und Ärzte

- Förderung der Teilnahme der Ärztinnen und Ärzte am Kurs/Workshop «Ausbildung der Ärzteschaft zur Tabakentwöhnung" (mit dem neuen Ausbildungsprogramm FMH/BAG "Frei von Tabak»). Nach Abschluss des Kurses erhalten die Teilnehmenden ein "Zertifikat für die Tabakentwöhnung».

- Förderung der Verteilung der «Richtlinien für die klinische Praxis" ("clinical practice guidelines»), denen zu entnehmen ist, wann die pharmakologische und nicht pharmakologische Unterstützung bei der Tabakentwöhnung indiziert ist.

Medizinstudentinnen und -studenten

Publikation der Resultate der beiden Studien über die Rauchenden in der Schweiz, z. B. in der Zeitschrift des Verbands Schweizerischer Assistenz- und Oberärzte/ -ärztinnen (VSA0).

Apothekerinnen und Apotheker

- Anregen der Ausbildung zur Tabakentwöhnungsberatung, z.B. das ursprünglich für die Ärzteschaft bestimmte Ausbildungsprogramm auf diese Berufsgruppe ausdehnen.

- Förderung der Verteilung von Broschüren zur Tabakentwöhnung (z.B. die Broschüre «Ziel Nichtrauchen") in den Apotheken.

Zahnärztinnen und Zahnärzte

- Anregen der Ausbildung zur Tabakentwöhnungsberatung, z.B. das ursprünglich für die Ärzteschaft bestimmte Ausbildungsprogramm auf diese Berufsgruppe ausdehnen.

- Förderung der Verteilung von Broschüren zur Tabakentwöhnung (z.B. die Broschüre «Ziel Nichtrauchen») in den Zahnarztpraxen.

Pflegepersonal/Physiotherapeutinnen und -therapeuten

- Ermutigung des Pflegepersonals bzw. der Physiotherapeutinnen und -therapeuten zur Aufgabe des Rauchens. In diesen Berufsgruppen ist die Prävalenz gleich hoch wie in der Allgemeinbevölkerung.
- Unterstützung von Ausbildungsprojekten zur Raucherentwöhnungsberatung, z.B. das ursprünglich für Ärzteschaft entwickelte Ausbildungsprogramm auf diese Berufsgruppe ausdehnen.

- Unterstützung der Verbreitung des Ausbildungsprogramms der Arbeitsgemeinschaft Tabakprävention für das nicht ärztliche Personal.

Auch Fachpersonen für die Tabakberatung könnten bei diesen Massnahmen miteinbezogen werden.

\section{Ziel 2}

Den Wandel der Verhaltensnormen bei der Schweizer Bevölkerung im Umgang mit dem Tabak unterstützen. Dieses Ziel versteht sich in Ergänzung zu Ziel 1.

\section{Allgemeine Massnahme}

Gesetzliche Regelung der Tabakprodukte, z.B. Verkaufsverbot für Zigaretten an Jugendliche unter 16 Jahren, Einschränkung (oder sogar Untersagen) der Werbung für Tabakprodukte.

\section{Spezifische Massnahmen}

- Breite Veröffentlichung der Studienresultate über die abhängig machenden Eigenschaften von Nikotin.

- Integration der medizinischen Gesellschaften (Kantonale Ärztegesellschaften und Medizinische Fachgesellschaften FMH) in den Prozess «Kontrolle des Tabakkonsums", z.B. indem man sie bittet, die in diesem Text aufgeführten Massnahmen zu unterstützen und die "Richtlinien für die klinische Praxis" zu verteilen.

- Integration der übrigen Gesundheitsberufe, z.B. die Berufsverbände der Krankenschwestern und Krankenpfleger und der Physiotherapeutinnen und -therapeuten in den Prozess "Kontrolle des Tabakkonsums", z.B. indem man sie ermutigt, die Hilfe zur Tabakentwöhnung als eine wichtige Aufgabe wahrzunehmen.

- Die Fachorganisationen (AT, Ligen) vermehrt beiziehen.

- Ausarbeiten von wissenschaftlichen Argumenten, die den Unwahrheiten der Zigarettenproduzenten systematisch entgegengesetzt werden können [23].

\section{Ziel 3}

Die Kostenübernahme durch die Krankenkassen für die Einzelberatung, die pharmakologische Unterstützung (Nikotinsubstitute, Bupropion) und die nicht pharmakologische Unterstützung (Einzel- oder Gruppensitzungen) zur Tabakentwöhnung durchsetzen.

Dieses Ziel versteht sich als Fortsetzung von Ziel 1 und 2. 


\section{Allgemeine Massnahme}

Veröffentlichung der wissenschaftlichen Daten über die Effizienz der Massnahmen zur Tabakentwöhnung, d.h. der Einzelberatung, der pharmakologischen Unterstützung (Nikotinsubstitute, Bupropion) und der nicht pharmakologischen Unterstützung (Einzeloder Gruppensitzungen).

\section{Spezifische Massnahmen}

- Auf die betroffenen Organisationen (Bundesamt für Sozialversicherungen, Konkordat der Schweizerischen Krankenversicherer) Druck ausüben, um folgendes zu erreichen:

- Einschluss in die krankenkassenpflichtigen Leistungen der «minimalen Intervention» für Rauchende im Stadium «Unentschiedenheit»;

- Durchsetzen der Kostenübernahme der Tabakentwöhnungsberatung (Rauchende in den Stadien Abwägen, Vorbereiten und Handeln) durch die Grundversicherung der Krankenkassen gemäss Art. 26 des KVG.

- Schaffen eines «Fähigkeitsausweis für die Tabakentwöhnung" zur Abgabe an das medizinische und nicht medizinische Personal (siehe Ziel 1).

\section{Ziel 4}

Entwicklung einer Methode zur langfristigen Erforschung der Prävalenz des Tabakkonsums und der tabakbedingten Probleme.

\section{Allgemeine Massnahme}

Anregen einer nationalen Zusammenarbeit bei Kohortenstudien, z.B. zwischen der Schweizerischen Gesellschaft für Prävention und Gesundheitswesen und den medizinischen Universitätspolikliniken.

\section{Spezifische Massnahmen}

- Nutzung dieser Zusammenarbeit für die Organisation von internationalen Treffen.

- Fortführung des «Massnahmepakets Tabak».

Die Expertengruppe unterstützt die verschiedenen bereits im Massnahmepaket enthaltenen Strategien, insbesondere:

- Besteuerung der Tabakprodukte.

- Partnerschaft/Sponsoring für Sport- und Freizeitaktivitäten für Jugendliche.

\section{Literatur}

1 Bundesamt für Statistik. Schweizerische Gesundheitsbefragung. Bern 1998;108/98.

2 Wyser C, Bolliger C. Smoking related disorders. In: Bolliger C, Fagerstrom K (eds.). The tobacco Epidemic. Basel: Karger; 1997. p. 78-107.
3 Doll R, Peto R, Weathtey K, Gray R, Sutherland I. Mortality in relation to smoking: 40 years of observations on male British doctors. Brit Med J 1994;309:901-11.

4 Department of Health and Human Services. The health benefits of smoking cessation. A report of the Surgeon General, 1990. DHHS publication No CDC-90-8416. Rockville: US Department of Health and Human Services; 1990.

5 Samet JM. The health benefits of smoking cessation In: Fiore MC (ed.). Cigarette smoking, A clinical guide to assessment and treatment. Philadelphia: Saunders; 1992. p. 399-14.

6 Cornuz J, Humair JP. L'arrêt du tabac: pourquoi, quand et comment? Med Hyg 2000;58:599-605.

7 Junker C, Töny G, Abelin T. Wunsch, Versuch und Erfolg, mit dem Rauchen aufzuhören. Ergebnisse einer Bevölkerungsbefragung in der deutschen Schweiz. Bern, Institut für Sozial- und Präventivmedizin; 1999.

8 Cornuz J, Wasem YM, Junker C, Abelin T. The use of stages of change model to tailor counseling for smokers in an outpatient clinic. Nicotine Tob Res 1999;1:190.

9 Prochaska JO, DiClemente CC. Stages and processes of selfchange in smoking: toward integrative models of change. J Consult Clin Psychol 1983;51:390-5.

10 Schmid H, Gmel G, Janin Jaquat B. Predictors of smoking status after eight years: the interaction of stages of change and addiction variables? Psychol Health 1999;14:731-46.

11 Humair JP, Sanchez-Menegay C, Stalder H. Prévention par les médecins de premier recours à Genève: attitudes, habitudes personnelles et pratiques cliniques. Communication orale. Assemblée Annuelle de la Société Suisse de Médecine Interne, Zürich. 1996.

12 Fiore MC, Novotny TE, Pierce JP, Giovino GA, Hatziandreu EJ, Newcomb PA, et al. Methods used to quit smoking in the United States: do cessation programs help? JAMA 1990; 263:2760-5.

13 Etter JF. Rapport d'évaluation du CIPRET-Genève. Genève: Université de Genève; 1996.

14 Kottke TE, Battista RN, DeFriese GH, Brekke ML. Attributes of successful smoking cessation interventions in medical practice. JAMA 1988;259:2882-9.

15 Law M, Tang JL. An analysis of the effectiveness of interventions intended to help people stop smoking. Arch Intern Med 1995;155:1933-41.

16 Silagy C, Ketteridge S. Physician advice for smoking cessation (Cochrane Review). In: The Cochrane Library. Issue 2. Oxford: Update Software; 1999.

17 Silagy C, Mant D, Fowler G, Lancaster T. Nicotine replacement therapy for smoking cessation (Cochrane Review). In: The Cochrane Library. Issue 4. Oxford: Update Software; 1999.

18 Cornuz J, Zellweger JP, Mounoud C, Decrey H, Pécoud A, Burnand B. Smoking cessation counseling by residents in an outpatient clinic. Prev Med 1997;26:292-6.

19 Hurt RD, Sachs DPL, Glover, Offord KP, Johnston JA, Dale LC, et al. A comparison of sustained-release Bupropion and placebo for smoking cessation. N Engl J Med 1997; 337:1195-202.

20 Fiore MC, Bailey MC, Cohen SJ, Dorfman SF, Goldstein MG, Gritz ER, et al. Treating tobacco use and dependence. Clinical practice guideline. Rockville, MD: Public Health Service; 2000 (AHRQ Publication No 00-0032)

21 Etter JF. Programme Stop-Tab@c. Genève: Institut de médecine sociale et préventive; 1998.

22 Abrams DB, Mills S, Bulger D. Challenges and future directions for tailored communication research. Ann Behav Med 1999;21:299-306.

23 Cornuz J, Burnand B, Kawachi I, Gutzwiller F, Paccaud F. Why did Swiss citizens refuse to ban tobacco advertising? Tob Control 1996;2:149-53. 